\title{
Learning curve of uniportal video-assisted lobectomy: analysis of 15-month experience in a single center
}

\author{
Dania Nachira ${ }^{1}$, Elisa Meacci ${ }^{1}$, Venanzio Porziella ${ }^{1}$, Maria Letizia Vita ${ }^{1}$, Maria Teresa Congedo ${ }^{1}$, Marco \\ Chiappetta $^{1}$, Leonardo Petracca Ciavarella ${ }^{1}$, Mahmoud Ismail ${ }^{2}$, Elisabetta Gualtieri ${ }^{3}$, Alfredo Cesario ${ }^{1}$, \\ Stefano Margaritora ${ }^{1}$
}

${ }^{1}$ Department of General Thoracic Surgery, Catholic University of Sacred Heart, Fondazione Policlinico Universitario “A.Gemelli”, Rome, Italy; ${ }^{2}$ Competence Center of Thoracic Surgery, Department of Surgery, Charité - Universitätsmedizin Berlin, Germany; ${ }^{3}$ Emergency Department, Catholic University of Sacred Heart, Fondazione Policlinico Universitario “A.Gemelli”, Rome, Italy

Contributions: (I) Conception and design: D Nachira, E Meacci, M Ismail; (II) Administrative support: S Margaritora, A Cesario ; (III) Provision of study materials or patients: V Porziella, ML Vita, MT Congedo, E Gualtieri; (IV) Collection and assembly of data: M Chiappetta, L Petracca Ciavarella; (V) Data analysis and interpretation: D Nachira; (VI) Manuscript writing: All authors; (VII) Final approval of manuscript: All authors.

Correspondence to: Dania Nachira, MD. Department of General Thoracic Surgery, Fondazione Policlinico Universitario “A.Gemelli”, Largo A. Gemelli, 8, 00168 Rome, Italy. Email: danynac@libero.it.

Background: Uniportal video-assisted thoracoscopic (U-VATS) lobectomy has been becoming the technique of choice in an increasing number of centers. The aim of our study was to review our experience, evaluating the learning curve of U-VATS for lung lobectomy and outcomes.

Methods: The prospectively collected clinical data of 43 consecutive patients, undergone U-VATS lobectomy from June 2016 to September 2017, were reviewed. The cumulative sum analysis was applied for defining the completion of learning curve (CLC), evaluating the relationship between operative time and the consecutive number of operations.

Results: The mean operative time of Uniportal VATS lobectomy was $179.93 \pm 43.41 \mathrm{~min}$. According to the cumulative sum analysis, the CLC was reached after 25 patients. Using the cut-off of 25 patients, the whole populations was divided in group A (first 25 patients of the experience) and group B (the last 18 patients). The mean operative time in group B was significantly shorter than in group A $(164.00 \pm 24.46 v s$. $191.40 \pm 50.45 \mathrm{~min}$, respectively, $\mathrm{P}=0.04$ ). There were no differences in demographic characteristics, number of removed lymph nodes, chest tube duration, and hospital stay among the two groups. The number of conversions was higher in group $\mathrm{A}$ ( 4 vs. $0 ; \mathrm{P}=0.07$ ), as the number of major complications, like reoperations for bleeding ( 2 ss. $0 ; \mathrm{P}=0.22$ ). There was no postoperative 30 -day-related death.

Conclusions: U-VATS lobectomy seems to be a quite safe and feasible procedure, with a steep learning curve and low complication rate, if performed by experienced surgeons after proper training.

Keywords: Uniportal video-assisted thoracoscopic (U-VATS); learning curve; lung cancer; lung major resections

Submitted Jan 28, 2018. Accepted for publication Mar 15, 2018.

doi: $10.21037 /$ jtd.2018.03.133

View this article at: http://dx.doi.org/10.21037/jtd.2018.03.133

\section{Introduction}

In the last decade, thanks to the great progresses of technologies and instrumentations and riding the wave of Diego Gonzales-Rivas $(1,2)$, an increasing number of centers have been choosing the minimally invasive uniportal video-assisted thoracoscopic (U-VATS) access for performing major lung resections.

A lot of efforts have been lavished for showing and teaching the technique all over the world as never before, 
thanks to the several masterclasses and dedicated courses organized by U-VATS surgeons.

Like any technique, U-VATS has its own rules and fundamental principles necessary for performing the surgical procedure in the easier and safer way. Therefore an appropriate learning curve has to be overcome before being proficient in U-VATS major lung resections. The purpose of these tutored courses is just that to provide everybody important and easy tricks for acquiring and mastering the technique, by experienced surgeons.

The aim of this work is to review the experience of a Thoracic Surgery center, with 15-month experience in U-VATS but after attending dedicated courses and masterclasses, and to evaluate the learning curve of U-VATS for lung lobectomy and outcomes.

\section{Methods}

The prospectively collected clinical data of patients undergone U-VATS lobectomy at Thoracic Surgery Department of Fondazione "A.Gemelli" University Hospital in Rome, were retrospectively reviewed.

Of 45 consecutive U-VATS lobectomies performed, from June 2016 to September 2017, 43 were suitable for the analysis, after excluding a case with strong adhesions requiring long adhesiolysis and a complex case with chest wall resection.

Being the present experience in U-VATS surgery an early one, the following criteria were respected for the selection of patients candidate for U-VATS lobectomy at our center: only cN0 or cN1 (without big lymphadenopathies) lung cancers, not central tumors, not previous induction therapy.

Preoperative assessments included blood analyses, electrocardiogram (with possible echocardiography or other cardiological examinations when required by preoperative evaluation of the anesthesiologist) chest tomography, PET-CT scan and lung function test. When feasible, the patients underwent a preoperative biopsy of the pulmonary lesion in order to confirm the neoplastic nature by bronchoscopy, in case of quite central lesions, or by CT-guided fine needle aspiration biopsy (FNAB), in case of peripheral ones. Endobronchial ultrasound, with FNAB when necessary, was also carried out preoperatively for confirming the clinical N-staging.

All patients signed an informed consent before the surgical operation for the treatment of their clinical data.

All standard U-VATS lobectomies with hilar-mediastinal lymphadenectomy performed by the same team were included in this study.

The performing surgical team had already a good experience in triportal, biportal VATS and robotic lobectomies. Indeed, in our center a VATS program for minor procedures was established more than 10 years ago (performing pulmonary wedge resections and resections of mediastinal lesions in Triportal VATS, while pleural biopsies, toilettes of pleural cavity and sympathectomies in single-access VATS), but only in 2013 the first Triportal VATS lobectomies (about the $16 \%$ of a mean of 150 VATS procedures per year) were performed. In the same year, we also started a robotic program (Da Vinci system), performing about 50 procedures per year (thymectomies, minor and major lung resections). Robotic lobectomies were about the $20 \%$ of the total procedures.

Since 2014, we continued the robotic program only for thymic surgery, considering thoracoscopy more convenient for lung surgery. Therefore, we started to perform minor and major (about the $30 \%$ of a mean of 180 VATS operations per year) lung resections in Biportal VATS. All pleural biopsies and sympathectomies were performed in single-access VATS as usual in our center. In May 2016 we introduced U-VATS program, that substituted biportal VATS, for lung surgery performing the first U-VATS minor lung resection and in June 2016 the first lobectomy.

All U-VATS lobectomies were performed in general anesthesia with double lumen ventilation. Patients were operated in lateral decubitus, with both surgeons (the operator and the assistant) standing on ventral side.

A $3-4 \mathrm{~cm}$ incision was performed on the anterior axillary line in the IV or $\mathrm{V}$ intercostal space, according to the localization of the lesion (3). A wound protector was always placed for preventing the soiling of the $30^{\circ} 10 \mathrm{~mm}$ camera, during its introduction in the posterior part of the incision, and contamination of the incision. The camera was always held and handled by the assistant surgeon, who had to know very well the different steps of the operation in order to provide the operator the best view of the target, show him in time any problem or critical situation for preventing errors and help him with lung tractions.

For patients that already had a preoperative diagnosis of NSCLC, after having confirmed intraoperatively the presence of the tumor in the lobe signaled by preoperative CT scan, the planned lobectomy could be performed. In case of lack of preoperative histological diagnosis, an intraoperative frozen section was carried out, before proceeding with lobectomy. Usually the time required for such analysis was about $40 \pm 10 \mathrm{~min}$ in our center; however, 
for any case, where a fresh frozen was executed, the overall operative time reported in the present paper have to be considered already reduced by the corresponding time necessary for the intraoperative examination.

It was always fundamental visualizing the hilum and checking the origin of the veins for each lobe, for excluding the presence of a common vein. After that each vascular structure for the target lobe was identified, dissected and cut by the use of an endostapler or clip and energy devices in case of small branches. The lobar bronchus was easily cut by stapler (a mean of 3 stapler reloads were used for cutting the main lobar bronchovascular structures per each lobe). Fissure was completed by stapler or sealing the parenchyma trough an energy device. Fissureless upper lobectomies were performed according to the fissure-last technique (a mean of 4-5 stapler reloads for parenchyma were used in these cases).

The operation was concluded by a systematic lymphadenectomy of all stations. The surgical specimens were removed by an Endobag.

For a better postoperative analgesia, an intrapleural paravertebral intercostal nerve block was performed, infiltrating ropivacaine in 3-4 intercostal spaces above and below the incision, under endoscopic view.

One 24 or $28 \mathrm{Fr}$ chest tube was inserted in the upper part of the incision and fixed to the skin (3).

Usually, the drain was removed when no air-leak sign was found and the secretion was below $200-250 \mathrm{~mL}$ within $24 \mathrm{~h}$.

\section{Data collection}

Preoperative clinical variables, like age, gender, smoking habits, comorbidities, neoadjuvant therapy, side of operation, histology and tumor size were evaluated.

Operative time and postoperative outcomes, like complications, conversion, chest tube duration and postoperative hospital stay were also collected and analyzed.

Mortality was defined as death occurring within 30 days after surgery.

\section{Statistical analysis}

Continuous variables were expressed as mean \pm standard deviation and compared using Student $t$-test. Categorical variables were analyzed using Fisher's exact or the chisquare test.

Pearson correlation coefficient was used to explore the type of correlation between the number of consecutive lobectomies and the corresponding operative time of the procedures.

The cumulative sum (CUSUM) technique was applied for defining the completion of learning curve (CLC) of U-VATS lobectomy, evaluating the relationship between operative time and the consecutive number of operations (4).

The CUSUM series was defined as follow: $\sum(\mathrm{Xi}-\mathrm{X} 0)$, where $\mathrm{Xi}$ was an individual measurement and $\mathrm{X} 0$ was a predetermined reference level and was set as the mean operative time for all the cases here.

The CUSUM series was plotted against the consecutive procedures. The point of downward inflection on the graph represented the cutoff value.

Subsequently, the cutoff point of CUSUM score was used to divide all patients in two groups: group A ( $\leq$ cutoff value) representing the early-experience group and group $B$ (> cutoff value) the late-experience one. The main outcomes of the two groups were also evaluated.

A $\mathrm{P}$ value less than 0.05 was considered statistically significant.

Statistical analysis was performed using PASW Statistics for Windows, Version 18.0 (SPSS Inc., Chicago, IL, USA).

\section{Results}

A total of 43 patients (21 males and 22 females) underwent U-VATS lobectomy; the average age was $68.30 \pm 9.39$ years.

The mean operative time was $179.93 \pm 43.41 \mathrm{~min}$ $(194.63 \pm 52.17 \mathrm{~min}$ for upper lobectomies, $167.86 \pm$ $25.78 \mathrm{~min}$ for middle lobectomies and $168.47 \pm 34.25 \mathrm{~min}$ for lower ones, with no significant statistical difference between mean operative times for upper and lower lobes, $\mathrm{P}=0.09$ ).

No difference in mean operation time was recorded between lobectomies with complete or incomplete fissure

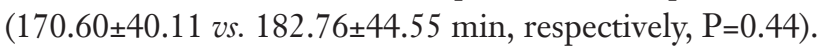

Thirty-eight $(88.4 \%)$ patients were operated on for a primary lung cancer; the mean dimension of the tumor was $2.69 \pm 1.52 \mathrm{~cm}$ and the number of lymph nodes removed was $13.40 \pm 9.96$.

The main characteristics of the series were summarized in Table 1.

In our experience, the length of operation and the consecutive number of procedures presented a statistically significant linear correlation $(y=-1.0532 x+203.0996)$. The Pearson correlation coefficient ( $\mathrm{r}$ ) was -0.305 with a twotailed $\mathrm{P}=0.04$ (Figure $1 A$ ).

It means that after 22 lobectomies the operative time was shorter than the mean operative time of all series. 
Table 1 Clinicopathological characteristics of patients of the entire cohort

\begin{tabular}{|c|c|}
\hline Variable & Total $(n=43)$ \\
\hline \multicolumn{2}{|l|}{ Preoperative characteristics } \\
\hline Age (years) & $68.30 \pm 9.39$ \\
\hline Gender (male) & $21(48.8 \%)$ \\
\hline Smoking & $13(30.2 \%)$ \\
\hline COPD & $17(39.5 \%)$ \\
\hline Hypertension & $28(65.1 \%)$ \\
\hline Cardiovascular diseases & $15(34.9 \%)$ \\
\hline ASA score & $2.35 \pm 0.48$ \\
\hline Neoadjuvant therapy & $0(0 \%)$ \\
\hline \multicolumn{2}{|l|}{ Intraoperative results } \\
\hline Primary lung cancer & $38(88.4 \%)$ \\
\hline Lesion dimension (cm) & $2.69 \pm 1.52$ \\
\hline Lymph nodes removed & $13.40 \pm 9.96$ \\
\hline Operative time (min) & $179.93 \pm 43.41$ \\
\hline Right side lobectomy & $34(79.1 \%)$ \\
\hline Upper lobectomy & $19(44.2 \%)$ \\
\hline Middle lobectomy & $7(16.3 \%)$ \\
\hline Lower lobectomy & $17(39.5 \%)$ \\
\hline Conversion & $4(9.3 \%)$ \\
\hline \multicolumn{2}{|l|}{ Postoperative outcomes } \\
\hline Reoperation for bleeding & $2(4.7 \%)$ \\
\hline Chest tube duration (days) & $5.90 \pm 4.97$ \\
\hline Hospital stay (days) & $5.51 \pm 2.55$ \\
\hline Minor complications & $10(23.3 \%)$ \\
\hline Thirty-day mortality & $0(0 \%)$ \\
\hline
\end{tabular}

COPD, chronic obstructive pulmonary disease; ASA, American Society of Anesthesiologists.

The CLC cut-off value was settled by the point of downward inflection on the plot of CUSUM analysis (Figure 1B), and was observed after 25 patients.

Using the CLC cut-off of 25 patients, the whole populations was divided in group A (first 25 patients of the experience) and group B (the last 18 patients).

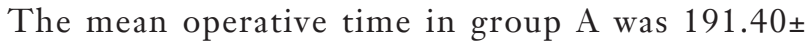
50.45 min while in group $B$ was $164.00 \pm 24.46$ min, $\mathrm{P}=0.040$.

There were no differences in demographic characteristics, number of removed lymph nodes, chest tube duration and hospital stay among the two groups (Table 2).

The number of conversions was $4(16.0 \%)$ in group A and 0 in group B, with a trend towards statistical significant $(\mathrm{P}=0.075)$. The reasons for conversion were technical difficulties in 3 cases $(12 \%)$ and a bronchial injury in 1 case (4\%).

Also the number of reoperations for non-major bleeding decreased from $2(8.0 \%)$ in group A to 0 (0) in group B $(\mathrm{P}=0.219)$. Instead the number of minor postoperative complications was quite the same: $5(20.0 \%)$ in group A vs. $5(27.8 \%)$ in group $\mathrm{B}(\mathrm{P}=0.551)$.

The main characteristics of both groups stratified by CLC cut-off are shown in Table 2.

The 30-day mortality was nil in both groups.

\section{Discussion}

As already widely described in literature, U-VATS for major lung resections is a safe and feasible technique $(1,2,5)$. In the last decades, the rapid development of the technique has allowed the execution of more complex procedures, like segmentectomies $(6,7)$ pneumonectomies $(8)$, vascular and bronchial sleeves (9).

Furthermore, U-VATS also seems to have more advantages for the patients compared to traditional VATS, like a faster postoperative recovery (10) and a less postoperative pain and paresthesia $(11,12)$. A series of benefits were also reported for surgeons in favor of U-VATS, like a direct visualization of the target like in open surgery, with a better eye-hand coordination $(13,14)$, a more ergonomic posture (15) and a potential faster training for master the technique.

In 2008, McKenna (16) suggested a length of learning curve for VATS lobectomy of at least 50 procedures. The same target was suggested by Petersen, that also underlined how several variables are involved in defining a learning curve (17). First of all the experience of the surgeon in open lobectomy by anterior approach and VATS surgery, the volume of major lung resections performed per year in the center, the type and stage of tumors operated and not last the individual differences and propensity towards a particular technique.

Some recent studies on U-VATS learning curve showed how U-VATS lobectomy can be performed safely by experienced surgeons, with a low risk of major complications (18) and a quite fast learning curve (19). Indeed, according to the Papworth group (19) after 30 out 
A

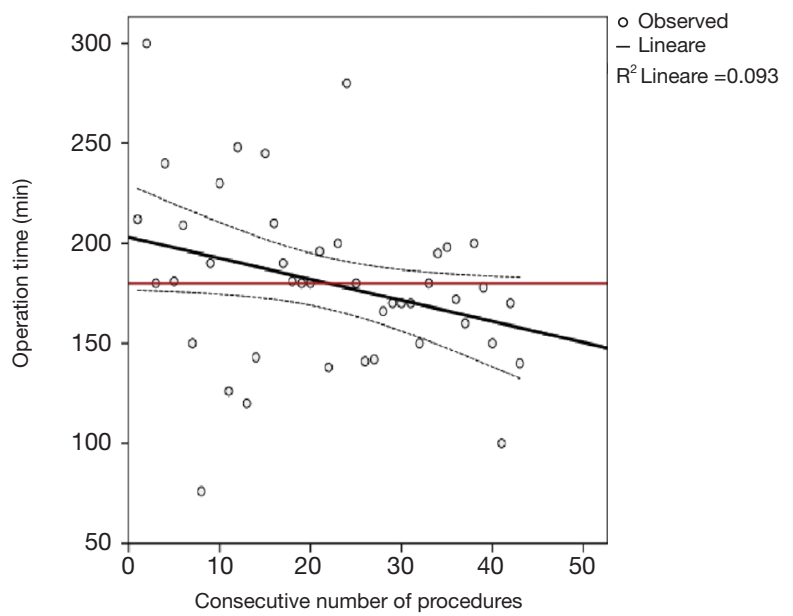

B

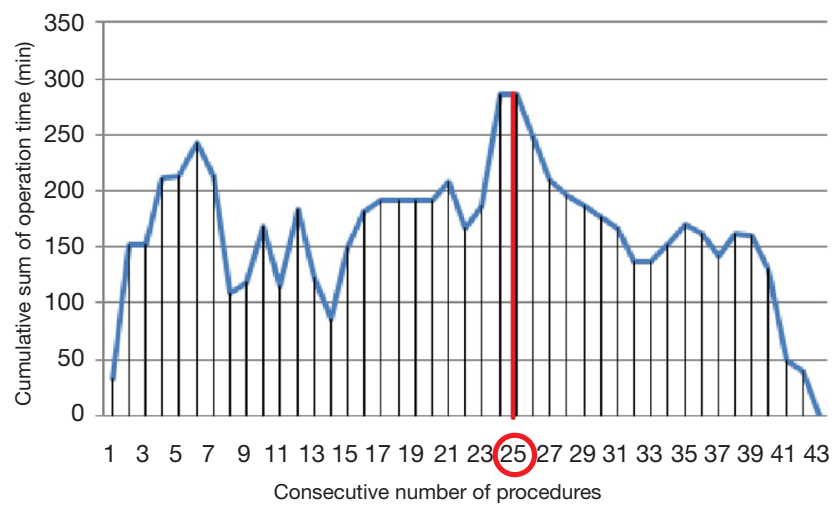

Figure 1 Learning curve of the first 43 U-VATS lobectomies. (A) Correlation between the operation time and the consecutive number of procedures (the red line represents the average time; the Pearson correlation coefficient (r) of linear function is -0.305 ); (B) cumulative sum (CUSUM) plot for the overall surgical time. The red circle is: CLC cut-off value on the plot of CUSUM analysis. U-VATS, uniportal videoassisted thoracoscopic; CLC, completion of learning curve.

of 73 lobectomies the established phase of learning curve was reached, with a significant reduction of complications, morbidity and mortality in the cases performed subsequently.

Comparing our results with those of Papworth group, with the limits related to the different type of analysis, we had a slightly faster learning curve ( 25 vs. 30 patients, respectively) and a lower conversion rate in the second phase of learning curve ( $0 \% v s$. $9.3 \%$, respectively). On the contrary, the Authors reported no reoperation for bleeding, while we had 2 cases (8\%) in the first phase. They also had a shorter hospital stay in their second phase of learning curve ( 3 days) compared to the first one ( 4 days, $\mathrm{P}=0.08$ ), in general inferior to our hospital stay that was about 5 days in both phases of our learning curve. This finding could be explained by the higher incidence of minor complications (like atrial fibrillation and postoperative air-leakage) that affected both groups of our patients in the postoperative period quite in the same proportion $(20 \%$ vs. $27.7 \%$, $\mathrm{P}=0.551)$.

The mean operative time in our series was $191.40 \pm$ $50.45 \mathrm{~min}$ in the first group and significantly lower $(164.00 \pm 24.46 \mathrm{~min})$ in the second one $(\mathrm{P}=0.04)$, including lobectomy and systematic lymphadenectomy of all stations; instead the Authors reported a not significant difference in time between the two groups $(84.9 \pm 33.0$ vs. $84.3 \pm$ $31.5 \mathrm{~min}, \mathrm{P}=\mathrm{NA}$ ) and it is not clarified in the manuscript if lymphadenectomy was included in that time.

The same results about the quite fast learning curve in U-VATS, were reported by Cheng and colleagues (20) for a more challenging and time-consuming procedure: the thoracoscopic segmentectomy. Their data indicated that after only 33 out of 40 U-VATS segmentectomies an improvement plateau was reached in their learning curve.

Talking about learning curve for Triportal VATS lobectomy, of the same opinion were Gezer and colleagues (21) in their manuscript, where CUSUM analysis reached the technical proficiency at 27 cases for duration of operations. In fact, they believe that, depending on the length of learning curve above all on previous experience of the surgeon, it is possible to obtain technical proficiency with an inferior number of procedures compared with the 50 or more cases of existing literature (16-18).

Their results for Triportal VATS lobectomy were comparable with ours for U-VATS (also for the same type of analysis) in terms of quite low number of cases for overcoming learning curve (27 vs. 25) and operative time (including lymphadenectomy); the postoperative hospital stay was shorter in our series $[5.51 \pm 2.55$ days $v s$. their 8.8 (range, 4-22) days] but in both series the incidence of minor postoperative complications was overlapping (23.2\% in our series vs. $22.4 \%$ in theirs).

According to the literature, also Robotic Assisted Thoracic Surgery (RATS) seems to have a quite steep 
Table 2 The clinicopathological characteristics of patients stratified by the completion of the learning curve (CLC) cutoff

\begin{tabular}{|c|c|c|c|}
\hline Variable & \multicolumn{2}{|c|}{ CLC cutoff } & $\mathrm{P}$ \\
\hline \multicolumn{4}{|l|}{ Preoperative characteristics } \\
\hline Age (years) & $68.44 \pm 7.70$ & $68.11 \pm 11.57$ & 0.911 \\
\hline Gender (male) & $11(44.0 \%)$ & $10(55.6 \%)$ & 0.455 \\
\hline COPD & 9 (36.0\%) & $8(44.4 \%)$ & 0.576 \\
\hline Hypertension & $16(64.0 \%)$ & $12(66.7 \%)$ & 0.856 \\
\hline Cardiovascular diseases & 8 (32.0\%) & 7 (38.9\%) & 0.640 \\
\hline ASA score & $2.24 \pm 0.44$ & $2.50 \pm 0.51$ & 0.081 \\
\hline Lesion dimension (cm) & $2.68 \pm 1.31$ & $2.70 \pm 1.86$ & 0.980 \\
\hline Lymph nodes removed & $12.90 \pm 9.06$ & $14.23 \pm 11.68$ & 0.710 \\
\hline Operative time (min) & $191.40 \pm 50.45$ & $164.00 \pm 24.46$ & 0.040 \\
\hline Right side lobectomy & $19(76.0 \%)$ & $15(83.3 \%)$ & 0.560 \\
\hline Upper lobectomy & $11(44.0 \%)$ & $4(22.2 \%)$ & 0.139 \\
\hline Middle lobectomy & $3(12.0 \%)$ & $4(22.2 \%)$ & 0.370 \\
\hline Lower lobectomy & $11(44.0 \%)$ & $10(55.6 \%)$ & 0.455 \\
\hline Conversion & $4(16.0 \%)$ & $0(0 \%)$ & 0.075 \\
\hline Minor complications & $5(20.0 \%)$ & $5(27.8 \%)$ & 0.551 \\
\hline Thirty-day mortality & $0(0 \%)$ & $0(0 \%)$ & _- \\
\hline
\end{tabular}

COPD, chronic obstructive pulmonary disease; ASA, American Society of Anesthesiologists.

learning curve for RATS lobectomy in expert hands, with about $18 \pm 3$ cases $(22,23)$.

Compared with Veronesi and colleagues' RATS experience (23), our results on U-VATS lobectomy showed shorter operative time $[191.40 \pm 50.45 \mathrm{~min}$ for the first 25 patients in our series vs. 260 (range, $152-$ 513) $\mathrm{min}$ for the first 18 patients in their series and $164.00 \pm$ $24.46 \mathrm{~min}$ in our second phase vs. 218 (range, 146-351) min in theirs], however in both series there was a statistically significant reduction of operative time from the first to the second phase of experience. We also recorded, in both phases of our U-VATS experience, a lower incidence of conversion (16\% and $0 \%$ vs. $17 \%$ and $10 \%$ of the authors), of complications (in particular we had the $8 \%$ of major complications (like bleeding) in the first phase and $0 \%$ in the second one $v s .11 \%$ and $4 \%$, respectively, reported by the authors) and a lower postoperative hospital stay $[5.32 \pm 1.95$ days and $5.78 \pm 3.24$ days, respectively in our 2 phases vs. 6 (range, 4-24) days and 5 (range, 3-23) days, in the 2 phases of authors' experience] compared with RATS. Furthermore, the number of lymph nodes retrieved in our U-VATS experience was quite overlapping with RATS 
one (23): $13.40 \pm 9.96$ vs. 15 (range, 4-30).

Therefore our results supported the safety and feasibility of Uniportal VATS lobectomy, with a quite steel learning curve. In a series of more than 200 U-VATS procedures in 15 months and 43 consecutive lobectomies, the cutoff of CUSUM score, applied for defining the completion of U-VATS lobectomy learning curve, was reached after 25 lobectomies.

After overcoming this step, all the outcomes showed a major proficiency of our team in performing Uniportal lobectomy. In particular, the operative time decreased significantly after the first 25 patients, with a less risk of conversion and postoperative complications.

Despite our wide experience in open lobectomies with muscle-sparing anterolateral thoracotomy, satisfactory experience in triportal, biportal VATS and robotic lobectomies, we believe in the importance of attending dedicated courses for improving the technique and shorten the learning curve.

In fact, like any technique, U-VATS has also its own rules (like the stapler angle, retraction technique and handle of the camera are different and require a different set of skills) and the precise knowledge and applications of such rules reduce the time-consuming in useless attempts and errors.

For this reason, we all attended dedicated U-VATS courses with Wetlab on animal models (24) before starting our U-VATS program, in particular each of two surgeons, that performed the whole series of lobectomies analyzed in this manuscript, attended at least two courses.

Furthermore, the main operator also spent a week visiting a high-volume center, observing U-VATS major lung resections and the assistant did a 3-month fellowship in a leading center for U-VATS surgery. During our U-VATS program, we also organized master classes (25), inviting experienced U-VATS surgeons for performing, scrubbed with us, supervised and mentored major resections (4 cases of the first U-VATS lobectomies performed in our center), learning important tips and tricks from them, directly during operation.

It was also extremely important selecting carefully the cases, standardizing the technique, above all during the learning curve, and operating always in two consultant surgeons. This approach, like also suggested by other authors (19), allowed to overcome technical difficulties, reduce surgical stress and ease the management of complications that could have required a conversion in the inexperienced hands.

This point could reopen the ongoing discussion about how the coming generations and trainees can learn to do
VATS lobectomies if the number of open procedures in VATS centers is reduced and dedicated to difficult cases, not suitable for VATS approach, and if first of all consultants have to overcome their learning curve.

But according to our experience, the adoption of a method like that we proposed, following the all above mentioned steps, gives the possibility to consultants to reach faster their proficiency in performing a U-VATS standard lobectomy and to start teaching residents first how to be a good assistant during U-VATS lobectomy and then how to operate as first surgeon. Meanwhile residents can observe and learn from their consultant's mistakes and train themselves in U-VATS technique, performing minor lung resections or assisting the operator in major procedures in simple steps.

And although experience learned from open surgery is fundamental, Okyere et al. (26) already stated-talking about multiportal VATS lobectomy-that the learning curve is not eliminated by prior experience in open lobectomy and young surgeons, with less experience in open lobectomy, can have similar outcomes and learning curves to more experienced surgeons.

The main limitations of our study are the small sample size and the monocentric model that results in a lack of comparison with other learning curves of U-VATS centers. Therefore a multicenter study would be claimed for the future.

In conclusion, U-VATS lobectomy seems to be a quite safe and feasible procedure, with a steep learning curve and low complication rate, if performed by experienced surgeons after proper training.

\section{Acknowledgements}

None.

\section{Footnote}

Conflicts of Interest: The authors have no conflicts of interest to declare.

Ethical Statement: This study was evaluated by the Institutional Review Board (IRB) of Catholic University of Sacred Hearth and, as this was a retrospective review for service evaluation (within an audit approved by our Surgical Department) and there was no modification in patients' care (no prospective randomized study), we did not need the final ethical approval of our IRB. 


\section{References}

1. Gonzalez D, Paradela M, Garcia J, et al. Single-port videoassisted thoracoscopic lobectomy. Interact Cardiovasc Thorac Surg 2011;12:514-5.

2. Gonzalez-Rivas D. VATS lobectomy: surgical evolution from conventional VATS to uniportal approach. ScientificWorldJournal 2012;2012:780842.

3. Ismail M, Swierzy M, Nachira D, et al. Uniportal videoassisted thoracic surgery for major lung resections: pitfalls, tips and tricks. J Thorac Dis 2017;9:885-97.

4. Cundy TP, Gattas NE, White AD, et al. Learning curve evaluation using cumulative summation analysis—a clinical example of pediatric robot-assisted laparoscopic pyeloplasty. J Pediatr Surg 2015;50:1368-73.

5. Ismail $M$, Helmig $M$, Swierzy $M$, et al. Uniportal VATS: the first German experience. J Thorac Dis 2014;6:S650-5.

6. Gonzalez-Rivas D. Single incision video-assisted thoracoscopic anatomic segmentectomy. Ann Cardiothorac Surg 2014;3:204-7.

7. Cheng K, Zheng B, Zhang S, et al. Feasibility and learning curve of uniportal video-assisted thoracoscopic segmentectomy. J Thorac Dis 2016;8:S229-34.

8. Gonzalez-Rivas D, Delgado M, Fieira E, et al. Uniportal video-assisted thoracoscopic pneumonectomy. J Thorac Dis 2013;5 Suppl 3:S246-52.

9. Gonzalez-Rivas D, Delgado M, Fieira E, et al. Double sleeve uniportal video-assisted thoracoscopic lobectomy for non-small cell lung cancer. Ann Cardiothorac Surg 2014;3:E2.

10. Ismail M, Swierzy M, Nachira D, et al. Fast-Tracking Patients Through the Diagnostic and Therapeutic Pathways of Intrathoracic Conditions: The Role of Uniportal Video-Assisted Thoracic Surgery. Thorac Surg Clin 2017;27:425-30.

11. Jutley RS, Khalil MW, Rocco G. Uniportal vs standard three-port VATS technique for spontaneous pneumothorax: comparison of post-operative pain and residual paraesthesia. Eur J Cardiothorac Surg 2005;28:43-6.

12. Louis SG, Gibson WG, King CL, et al. Uniportal videoassisted thoracoscopic surgery (VATS) technique is associated with decreased narcotic usage over traditional VATS lobectomy. J Vis Surg 2017;3:117.

13. Bertolaccini L, Rocco G, Viti A, et al. Geometrical characteristics of uniportal VATS. J Thorac Dis 2013;5 Suppl 3:S214-6.

14. Bertolaccini L, Rocco G, Pardolesi A, et al. The Geometric and Ergonomic Appeal of Uniportal
Video-Assisted Thoracic Surgery. Thorac Surg Clin 2017;27:331-8.

15. Bertolaccini L, Viti A, Terzi A. Ergon-trial: ergonomic evaluation of single-port access versus three-port access video-assisted thoracic surgery. Surg Endosc 2015;29:2934-40.

16. McKenna RJ Jr. Complications and learning curves for video-assisted thoracic surgery lobectomy. Thorac Surg Clin 2008;18:275-80.

17. Petersen RH, Hansen HJ. Learning curve associated with VATS lobectomy. Ann Cardiothorac Surg 2012;1:47-50.

18. Drevet G, Ugalde Figueroa P. Uniportal video-assisted thoracoscopic surgery: safety, efficacy and learning curve during the first 250 cases in Quebec, Canada. Ann Cardiothorac Surg 2016;5:100-6.

19. Bedetti B, Bertolaccini L, Solli P, et al. Learning curve and established phase for uniportal VATS lobectomies: the Papworth experience. J Thorac Dis 2017;9:138-42.

20. Cheng K, Zheng B, Zhang S, et al. Feasibility and learning curve of uniportal video-assisted thoracoscopic segmentectomy. J Thorac Dis 2016;8:S229-34.

21. Gezer S, Avci A, Türktan M. Cusum analysis for learning curve of videothoracoscopic lobectomy. Open Med (Wars) 2016;11:574-7.

22. Meyer M, Gharagozloo F, Tempesta B, et al. The learning curve of robotic lobectomy. Int J Med Robot 2012;8:448-52.

23. Veronesi G, Agoglia BG, Melfi F, et al. Experience with Robotic Lobectomy for lung cancer. Innovations (Phila) 2011;6:355-60.

24. Bedetti B, Schnorr P, Schmidt J, et al. The role of wet lab in thoracic surgery. J Vis Surg 2017;3:61.

25. Meacci E, Nachira D, Congedo MT, et al. Teaching uniportal video-assisted thoracic surgery in Rome. J Vis Surg 2017;3:49.

26. Okyere S, Attia R, Toufektzian L, et al. Is the learning curve for video-assisted thoracoscopic lobectomy affected by prior experience in open lobectomy? Interact Cardiovasc Thorac Surg 2015;21:108-12.

Cite this article as: Nachira D, Meacci E, Porziella V, Vita ML, Congedo MT, Chiappetta M, Petracca Ciavarella L, Ismail M, Gualtieri E, Cesario A, Margaritora S. Learning curve of uniportal video-assisted lobectomy: analysis of 15 -month experience in a single center. J Thorac Dis 2018;10(Suppl 31):S3662-S3669. doi: 10.21037/jtd.2018.03.133 